\section{Duloxetin bei Stressinkontinenz - mehr Schaden als Nutzen?}

\section{Eine Analyse individueller Patientendaten aus klinischen Studien stellt den Nutzen von Duloxetin bei Stressinkontinenz erneut infrage.}

E in Team um Dr. Emma Maund vom dänischen Cochrane Centre in Kopenhagen prüfte in einer Metaanalyse, ob bei einer Duloxetintherapie gegen Stressinkontinenz tatsächlich der Nutzen überwiegt. Dazu schauten sie sich EMA(European Medicines Agency)-Berichte zu vier bei der Agentur eingereichten placebokontrollierten Studien genauer an.

Während Duloxetin in Deutschland zur Therapie mittelschwerer bis schwerer Belastungsinkontinenzen bei Frauen angeboten wird, ist der Serotonin-/Noradrenalin-Wiederaufnahmehemmer zur Behandlung der Stressinkontinenz in den USA nicht zugelassen. Grund war eine erhöhte Rate von Suizidversuchen in Extensionsstudien. Da Duloxetin Stressinkontinenz-Patienten überaktivieren könnte, wird befürchtet, dass diese dann vermehrt zu Suiziden oder anderen Gewalttaten neigen. Das Team um Maund fahndete daher gezielt nach Symptomen, die ein solches Verhalten fördern könnten oder Gewalttaten vorausgehen, etwa innere Unruhe, Insomnie, Angst oder Aggressivität.

In den vier Studien hatten 958 Frauen Duloxetin $(80 \mathrm{mg} / \mathrm{d})$ und 955 Placebo erhalten; das Alter lag im Schnitt bei 52 Jahren. Patienten unter Antidepressiva wurden ausgeschlossen, auch solche mit Substanzmissbrauch; ansonsten konnten psychisch Kranke teilnehmen. Komorbiditäten, auch psychische, waren in den Studienarmen einigermaßen gleich verteilt, lediglich in einer Studie fanden sich im Placeboarm etwas mehr Patientinnen mit einer Depression in der Vergangenheit.

Im Schnitt hatten die Frauen zu Studienbeginn knapp 17 Inkontinenzepisoden pro Woche. In den einzelnen Studien ging die Zahl um zwei bis acht Episoden zurück, unter Duloxetin traten im Schnitt 2,9 Episoden weniger auf als mit Placebo. Relativ betrachtet lag die Differenz bei $14 \%$, die Unterschiede waren statistisch signifikant. Als klinisch relevant gilt eine Reduktion von mindestens 2,5 Episoden. Da das $95 \%$-Konfidenzintervall diesen Wert unterschritt, sei eine klinische Relevanz zumindest fraglich, urteilten die Cochrane-Analysten. Auch die Effektstärken waren ihrer Ansicht nach nicht eindrucksvoll (standardisierte Mittelwertdifferenz 0,1-0,3).

Unerwünschte Wirkungen traten insgesamt bei 727 Patientinnen mit Duloxetin und bei 548 mit Placebo auf. Aus fördernde Symptome entwickeln. diesen Angaben berechneten die Wissenschaftler um Maund eine „number needed to harm" (NNH) von 7. Um einen Therapieerfolg zu erzielen, müssen folglich acht Patientinnen behandelt werden („number needed to treat“, NNT), ein Schaden tritt aber bereits bei 7 auf, was für ein eher ungünstiges Nutzen-Risiko-Verhältnis spricht. Betrachteten die Forscher um Maund die Rate der Therapieabbrüche aufgrund unerwünschter Wirkungen, so war diese unter Duloxetin fünffach höher als unter Placebo. Auch hierfür berechneten sie eine NNH von 7. Suizide und andere Gewalttaten wurden bei keiner der Patientinnen beobachtet - hierfür war die Teilnehmerzahl sicher zu gering. Bei zwei Frauen unter Duloxetin traten jedoch starke unerwünschte Wirkungen auf, die Suiziden oft vorausgehen. Eine Überaktivierung wurde bei 187 Patientinnen mit Duloxetin und 42 unter Placebo beobachtet. Gewertet wurden hier etwa Insomnie, Agitation, Zittern oder Unruhe. Die NNH für solche Ereignisse lag ebenfalls bei 7. Emotionale Störungen traten unter Duloxetin ebenfalls gehäuft auf (18 vs. 3 Frauen), auch psychotische oder potenziell psychotische Erlebnisse (21 vs. 9 Frauen).

Fazit: „Diese Resultate legen nahe, dass der Nutzen von Duloxetin bei Stressin-

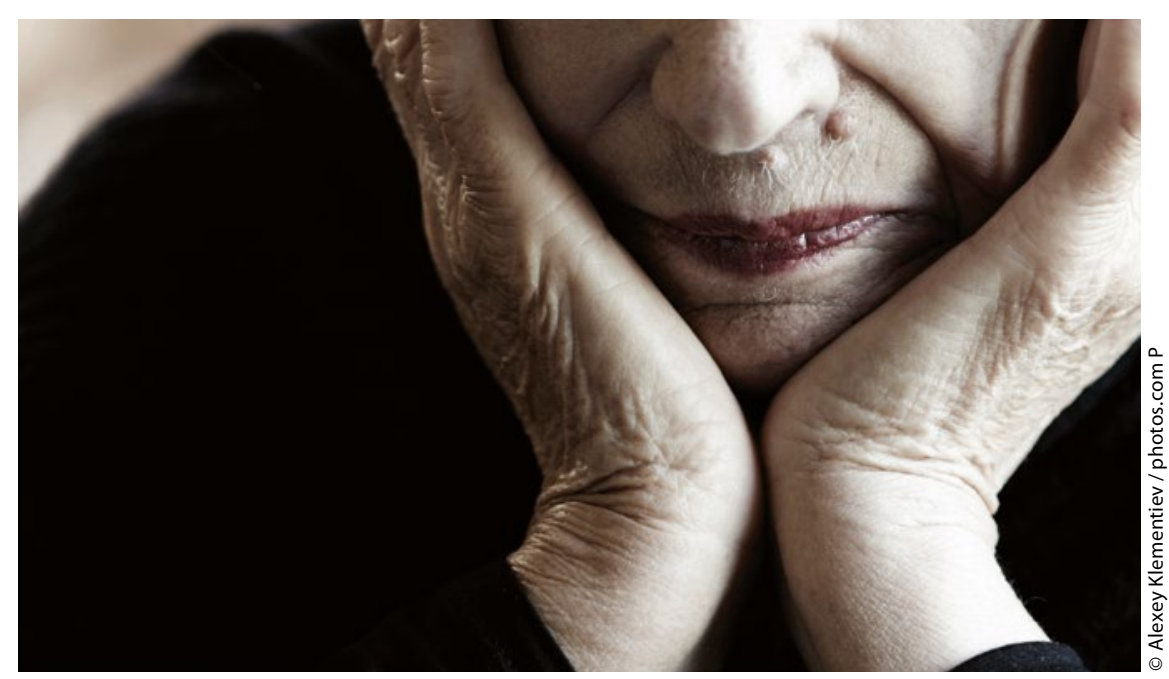

Patientinnen mit Stressinkontinenz können bei der Therapie mit Duloxetin suizid-

kontinenz nicht den Schaden überwiegt", schlussfolgern die Autoren der Analyse. Ferner hätten viele Patienten unter der Medikation unangenehme Erlebnisse, die Suizide oder Gewalttaten begünstigen könnten, schreiben sie und betonen: „Wir stellen daher die Rationale für Duloxetin bei Stressinkontinenz infrage.“

Thomas Müller

Maund $\mathrm{E}$ et al. Considering benefits and harms of duloxetine for treatment of stress urinary incontinence: a meta-analysis of clinical study reports. CMAJ. 2016; doi: 10.1503/cmaj.151104. 